\title{
Elicitation and the dominant character of stimulation-bound escape behavior*
}

\author{
JAN W. KAKOLEWSKI \\ Department of Physiology and Pharmacology \\ The University of South Dakota School of Medicine, Vermillion, South Dakota 57069
}

and

THOMAS C. ROWE

University of Wisconsin, Stevens Point, Wisconsin 54481

\begin{abstract}
Two groups of rats were implanted with bipolar electrodes in the medial forebrain bundle (MFB). Intracranial stimulation (ICS) was set to a minimal level, inducing continuous locomotion. When animals in Group I were mechanically restrained and ICS continued, all animals displayed a stimulation-bound escape behavior. Group II, prior to restraint, was exposed to ICS, eliciting continuous locomotion until stimulation-bound eating, drinking, or gnawing was established. When restrained, again, all animals displayed a consistent stimulation-bound escape behavior but not stimulation-bound eating, drinking, or gnawing. The data indicate that the combination of an internal with an environmental stimulus triggers a potent and highly consistent response dominating all previously established responses to ICS.
\end{abstract}

Long trains (usually more than $10 \mathrm{sec}$ ) of intracranial stimulation (ICS) applied to the lateral hypothalamus in the rat may elicit goal-oriented behavior such as eating, drinking, hoarding, etc. Since the behavior is present mostly during the period of stimulation, the term stimulation-bound behavior (SBB) has been applied (see, for review, Valenstein et al, 1970). During the course of inducting of SBB, it is a common practice to initially set the ICS current level at a point that elicits continuous locomotion of an exploratory character. When SBB is established, locomotion subsides at the time the rat engages in a goal-oriented behavior. Removal of the goal object(s) reinstates locomotion. With the location of the electrodes in the MFB, it is relatively easy to establish locomotion in the majority of stimulated animals. However, only less than half of the animals responding with locomotion might, upon further ICS, display stimulation-bound eating, drinking, or gnawing. The later behaviors develop gradually and might suppress the locomotion or alternate with locomotion, and are elicited without changes in the intensity of ICS.

Considering the apparent close relationship between SBB and locomotion, it was of interest to establish (1) whether suppression of locomotion by mechanical restraint would generate a behavior consistent with the environmental arrangement in Ss where SBB had not yet been established, and (2) whether such restraint would lead to a disruption of an established SBB, perhaps by supplanting it with another behavior. Since it is known that frequency of avoidance or escape increases with a rise in the intensity of ICS (Beer et al, 1968), the

\footnotetext{
*Supported in part by a grant from the Research Committee of the Medical School in Vermillion. Thanks are extended to Pearl Miller for histology, Vivian Johnson for preparation of the manuscript, and Daniel and Emily Kakolewski for encouragement.
}

intensity of ICS which elicited locomotion or SBB was kept constant throughout all experimental manipulations.

\section{METHODS, PROCEDURES, AND RESULTS}

The Ss were 12 mature Holtzman rats. Under Nembutal anesthesia, each animal was implanted with two bipolar electrodes bare of insulation at the cross-section of the tips. With the dorsal surface of the skull parallel to the horizontal, the electrodes were positioned $3.0 \mathrm{~mm}$ posterior to bregma, $1.7 \mathrm{~mm}$ lateral, and $8.5 \mathrm{~mm}$ below the top of the skull so as to intersect the lateral hypothalamic area.

At the end of the experiment, the animals were sacrificed, perfused with saline and Formalin, and histological verification of the placement of the tips performed. All tips were placed in the medial forebrain bundle at the level of the posterior edge of the lateral hypothalamic area.

The current used throughout was $60-\mathrm{Hz}$ sine wave, with the microampere level individually adjusted for each animal at the lowest level yielding continuous locomotion during $30 \mathrm{sec}$ of ICS. Once a value was found for a given animal, it was never changed throughout the entire experiment. The $30-\mathrm{sec} \mathrm{ON}$, 30-sec OFF pattern was continued for all phases of training and testing. The experimental Plexiglas chamber contained at all times water, Purina pellets, and a wedge of wood.

Two weeks following surgery, the animals were exposed to ICS while adjusting the current level, until continuous locomotion was elicited. At this point, the Ss were returned to their home cages.

The following day, six rats in Group I were mechanically restrained by being wrapped tightly with a thick, soft cotton cloth in a manner which excluded effective postural control and exploratory bodily movements. The head was not prevented from masticatory or orienting movements. The animal was laid on its abdomen and given 2 min to adapt; during this time, the animal tended to subside from its initial escape attempts. Food, water, and wood were within reach. At this point, ICS was initiated.

With the initiation of the first train, all six animals displayed highly vigorous movements, frequently accompanied by 
squealing and biting of the grid floor; the motoric excitement was exclusively confined to the period of stimulation, the animal becoming quiescent during the $30-$ sec OFF period. During subsequent trains, the excitement returned with similar intensity; in some instances, however, the excitement continued during the first $5-15 \mathrm{sec}$ of the OFF periods, but the intensity was markedly diminished.

The escape behavior generated by the interaction of the ICS and restraint was very effective, as illustrated by the time we were able to maintain the restraint (two rats for the full $20 \mathrm{~min}$, two for $15 \mathrm{~min}$, and two for $10 \mathrm{~min}$ ). Furthermore, all animals managed to injure themselves significantly in their struggles. These injuries usually involved reopening of the scalp wound from surgery and tearing of claws.

For the animals that freed them selves before the $20 \mathrm{~min}$ were up, the wrapping was removed from the cage and stimulation was continued. For the other two animals, stimulation was halted while the animals were freed by the $\mathrm{E}$, and then resumed. The free-moving animals displayed again the usual locomotion in response to ICS. The ICS was continued for $2 \mathrm{~h}$ per day for 6 days or until criterion on a different SBB than escape was reached. Criterion was defined as engaging in drinking, eating, or gnawing for 15 consecutive ICS trials. Of the six animals of Group I, only one displayed stimulation-bound eating. This rat was one of those restrained for only $10 \mathrm{~min}$, and it met criterion after $5 \mathrm{~h}$ of stimulation.

To establish whether the stimulation-bound escape would override a previously established goal-oriented behavior, six rats in Group II received ICS for $2 \mathrm{~h}$ per day following the current setting, until criterion on stimulation-bound eating, drinking, or gnawing was reached. Of these six, five met criterion with an average latency of $38 \mathrm{~min}$, maximum of $93 \mathrm{~min}$ (two gnawing, two drinking, one eating). One animal failed. On the day subsequent to meeting criterion, all animals were restrained in the same manner as the animals of Group I.

Restraint for Group II yielded much the same result as restraint for Group I in that there was severe struggling accompanied by significant injury and escape. Three of these Ss were restrained for the full $20 \mathrm{~min}$, two for $8 \mathrm{~min}$, one for $6 \mathrm{~min}$. No animal engaged in stimulation-bound eating, drinking, or wood gnawing. Following restraint, the ICS was continued and latency to SBB was measured. The five Ss originally displaying stimulation-bound gnawing, drinking, or eating returned to criterion on the same SBB with a maximum latency of $3 \mathrm{~min}$. The sixth $\mathrm{S}$ continued to display only locomotion.

On the possibility that the restraint itself was overly severe, a group of four unoperated rats was treated for the effects of restraint on water consumption. Two of these rats were water deprived for $24 \mathrm{~h}$, two for $48 \mathrm{~h}$. Following deprivation, the rats were restrained in the same manner as previously noted and placed with the head within $2.5 \mathrm{~cm}$ of the water delivery tube. The two deprived for $24 \mathrm{~h}$ explored the water spout, but did not ingest any significant amount of water. However, the two rats that were deprived for $48 \mathrm{~h}$ drank 7 and $8 \mathrm{ml}$ of water, respectively, with a latency of less than $1 \mathrm{~min}$. The drinking continued with occasional interruptions over the entire exposure time of $10 \mathrm{~min}$. On the second day of the procedure, the $24-\mathrm{h}$ water-deprived rats also ingested a normal load of water. A similar control for eating yielded comparable results, indicating that the restraint did not interfere with consumption.

\section{DISCUSSION}

Stimulation of the MFB with current intensity identical to the levels used for elicitation of locomotion or SBB yielded in all animals, when restrained, a stimulation-bound escape. Thus, it is possible to produce consistently one type of SBB, when environmental conditions are properly arranged. Furthermore, under conditions of restraint, locomotion and other SBB patterns established prior to restraint yielded to stimulation-bound escape and reappeared with short latency following removal of restraint. As noted, no change in ICS intensity was necessary.

Experimental and environmental arrangements, such as shuttlebox self-stimulation with continuous access to current, may elicit consistent object carrying in rats (Phillips et al, 1969). According to Mendelson and Maul (1973), the consistency of stimulation-bound object carrying can be varied in that its likelihood is higher when high intensities eliciting a "more immediate aversive neural process" are employed. In the present experiment, the intensity was not varied; it is possible that decreasing the current would eliminate stimulation-bound escape, but it is equally possible that locomotion and SBB would disappear, since to begin with these behaviors were established at the lowest possible level of current. Such speculations do not subtract from the fact that the stimulation-bound escape occurred in all animals at all trials, with apparently similar vigor, and depended exclusively upon restraint.

The escape behavior occurring during ICS in restrained rats is of significant contrast to the behavior elicited by ICS in freely moving animals; as a matter of fact, its intensity surpasses any escape form known to the present observers. However, difficulties in design, unavoidable in these types of studies, made these experiments descriptive rather than quantitative. Hence, the times provided for the illustration of our success in maintaining the restraint are distorted by the possible, but not noticeable, differences in the firmness of the restraint. Many attempts to design a different and effective mechanical technique failed in our hands.

\section{REFERENCES}

Beer, B., Steiner, S., \& Shaffer, M. M. The aversive properties of hypothalamic stimulation. Communications in Behavioral Biology, 1968, No. 5, Part B1. Abstract No. 05681162.

Mendelson, J., \& Maul, G. Effects of hypothalamic current intensity on object carrying induced by shuttlebox self-stimulation in rats. Physiological Psychology, 1973, 1, 181-184.

Phillips, A. G., Cox, V. C., Kakolewski, J. W., \& Valenstein, E. S. Object carrying by rats: An approach to the behavior produced by stimulation. Science, 1969, 166, 903-905.

Valenstein, E. S., Cox, V. C., \& Kakolewski, J. W. Reexamination of the role of the hypothalamus in motivation. Psychological Review, 1970, 77, 16-31.

Valenstein, E. S., Cox, V. C., \& Kakolewski, J. W. Reexamination of the role of the hypothalamus in motivation. In D. Singh and C. T. Morgan (Eds.), Current status of physiological psychology: Readings. Monterey, Calif: Brooks/Cole, 1972. Pp. 129-139.

(Received for publication November 29, 1973; revision received March 12,1974 .) 